\title{
Introduction to the Special Issue: 2020 Pacific Rim New Horizons in Human Brain Imaging: Neuroimaging across the Lifespan
}

\author{
Neda Jahanshad ${ }^{1}(1) \cdot$ Xi-Nian Zuo ${ }^{2,3}$ \\ Accepted: 16 November 2021 / Published online: 5 January 2022 \\ (c) The Author(s), under exclusive licence to Springer Science+Business Media, LLC, part of Springer Nature 2021
}

The human brain is dynamic in nature, and, for that matter, in nurture. The brain develops rapidly early in life, and continues to mature well into early adulthood, with changes continuing throughout life. While an individual's genetic makeup may frame the initial architecture of the brain, epigenetics, exposures, experiences, and other environmental factors are continuously integrated to shape how the brain develops, declines, and functions throughout life.

Deviation from healthy function is often indicative of an underlying disorder or disease, which can be brought on through injury or through seemingly organic processes, and these processes may have differential effects and consequences throughout the lifespan. Yet clinically observable changes in neurological and psychiatric function may only be detectable long after vulnerabilities brought on by structural and pathological degenerations have set the stage for decline.

The complexities of the human brain - and its unique susceptibility to individual experiences and risk factors-have made it arguably the most difficult organ to understand. The rate of scientific advancement in neuroscience has lagged and, as an unfortunate consequence, brain disorders are rapidly becoming a consistent top cause of morbidity and mortality around the world.

Brain imaging offers objective assessments of brain structure and function, and allows us to quantify processes that may not be otherwise observable. Technical advances

Neda Jahanshad

neda.jahanshad@usc.edu

1 Laboratory of Brain eScience; Mark and Mary Stevens Neuroimaging and Informatics Institute, Keck School of Medicine of USC, University of Southern California, Marina del Rey, CA 90292, USA

2 Developmental Population Neuroscience Research Center, IDG/McGovern Institute for Brain Research, State Key Laboratory of Cognitive Neuroscience and Learning, Beijing Normal University, Beijing 100875, China

3 National Basic Science Data Center, Beijing 100190, China in neuroimaging acquisition protocols and processing streams - especially in our current age of big data, structured information, collaboration, and computational power-provides the foundational support needed to reliably gain significant, and reproducible, insights into the living brain.

In 2020, the theme of the Pacific Rim New Horizons in Human Brain Imaging conference was Neuroimaging across the Lifespan. Here, renowned experts from around the world met with rising leaders and early-stage investigators to present current progress, novel discoveries, and budding initiatives. This discussion-heavy meeting highlighted the future directions necessary to understand the brain at every stage of life, and the challenges that need to be met in order to bridge and integrate the accumulated knowledge of brain function across all life stages.

In this Special Issue, we highlight ten original research reports led by several meeting attendees. In aggregate, they exemplify the types of neuroimaging research needed to gain critical insights into the human brain throughout life. A selection of work presented in this issue provides new insights from large-scale population studies and datasets, many of which have recently been made publicly available. Other works highlight understudied clinical populations and the changes in brain structure and function that occur with time. This issue uniquely integrates a variety of brain imaging modalities together with unique study designs or methods that together are essential for mapping individual and population-level trajectories of brain structure and function across the lifespan.

Hatoum et al., in their work entitled, Brain Structure and Problematic Alcohol Use: A Test of Plausible Causation Using Latent Causal Variable Analysis, use methods from statistical genomics along with available genome-wide summary statistics of problematic alcohol use and brain structure, from tens of thousands of individuals, to determine the extent to which variation in one trait is potentially genetically causal for the variation in other traits. The authors report that brain structure-specifically the tendency to have 
higher volume in the pars opercularis, thicker thickness in the cuneus, and smaller basal forebrain volume-may genetically contribute to problematic alcohol use. This implies that some differences in brain structure often reported in groups of problematic alcohol users relative to non-users are in part a predisposition risk factor for alcohol use, not purely a result of the alcohol use itself. This work highlights how publicly accessible resources, including published quantitative results, and methodological advances in statistical genetics can be used to suggest causal relationships between brain imaging derived traits and behavior, even in the absence of longitudinal data.

Luo et al. use a parent-offspring study design to also assess effects of predisposing risk factors on brain imaging traits. Focusing on the neurobiology underlying familial risk for childhood obesity, they examine how maternal BMI may predict the offspring's brain reactivity to food cues as determined by BOLD fMRI. In their work entitled, The Role of Maternal BMI on Brain Food Cue Reactivity in Children: A Preliminary Study, Luo and colleagues assessed the relationship between parental BMI and BOLD response changes in the dorsolateral prefrontal cortex (dIPFC) and the anterior cingulate cortex (ACC) of fasting children before and after a glucose ingestion. While they found no evidence to support a relationship to paternal BMI, current maternal BMI was negatively associated with food cue reactivity after glucose ingestion in both the dIPFC and the ACC, two regions for which higher BOLD response has been frequently linked with dietary self-control and eating behaviors. The offspring's brain reactivity was more strongly associated with current maternal BMI than with maternal pre-pregnancy BMI, despite a correlation between the BMI at these two time points. Such studies that assess cross-generational relationships between traits, such as BMI, and brain response, can provide added insights into how a complex combination of genetic and environmental risk factors installed in one generation may influence the cognitive and behavioral risk for their future generations.

Dewey et al. further our understanding of the developing brain by assessing structural brain correlates of a relatively common, but understudied developmental condition - developmental coordination disorder (DCD), a disorder characterized by motor deficits, specifically slow or inaccurate movements, that is often comorbid with attention deficit hyperactivity disorder (ADHD). In their study, Subcortical Brain Structure in Children with Developmental Coordination Disorder: A T1-Weighted Volumetric Study, Dewey and colleagues conduct a two-site volumetric analysis of brain structure in children with DCD and well-matched controls without DCD. They find preliminary evidence of smaller pallidum volumes in DCD children compared to typically developing controls, on average; yet they find no evidence to support DCD-related differences reported in cortical or cerebellar structures in other studies. They highlight inconsistencies in findings from the few neuroimaging studies of children with DCD and stress the need for coordination and collaboration across neuroimaging initiatives to address the paucity of studies focusing on DCD. The authors call for large-scale efforts to overcome the heterogeneity across samples, and gain substantial statistical power in order to converge on consistent and reproducible neuroimaging signatures of brain maturation with DCD, a disorder that interferes with the daily functioning of one in twenty children.

Brain maturation trajectories are extremely variable across the population. Yet regardless of the trajectory, brain development can be severely interrupted and subsequently altered by traumatic brain injury (TBI) - leading to chronic and potentially disabling consequences. In their work entitled White Matter Changes Following Experimental Pediatric Traumatic Brain Injury: An Advanced DiffusionWeighted Imaging Investigation, Zamani and coauthors use mouse models of early pediatric TBI (pTBI) to explore the sensitivity of advanced diffusion MRI metrics for quantifying these chronic developmental alterations in the white matter microstructure, months after the initial insult. To characterize the disruption in the structural connectivity profile after pTBI, this work scanned animal models with multishell high angular resolution diffusion imaging to reconstruct detailed profiles of diffusion at every voxel. This work models fiber orientations and associated streamlines, deriving quantitative metrics of tract-weighted imaging (TWI) along streamlines, voxelwise along each fiber direction, and more common diffusion models and corresponding microstructural metrics. The authors find that chronic injury after the initial insult was apparent in major white matter bundles, including the corpus callosum, using both standard and advanced diffusion modeling methods. However, the more advanced diffusion models were more sensitive to the differences between the pTBI and sham groups, even able to detect differences in the contralateral hemisphere. This work suggests such advanced diffusion imaging and beyond-tensor analyses of diffusion MRI may be a promising clinical tool to help uncover more subtle differences in chronic white matter injury during brain development.

Advanced diffusion MRI metrics can also be important for understanding normal variability throughout the lifespan. In Frontoinsular cortical microstructure is linked to life satisfaction in young adulthood, Cabeen et al. used advanced diffusion MRI metrics to identify brain correlates of life satisfaction in a young adult population from the Human Connectome Project. While most studies using diffusion MRI have often focused on quantifying white matter microstructure, authors here use multicompartmental models of diffusion to assess the microstructural properties of cortical gray matter, which may reflect an aggregation of cell bodies, myelination, dendritic complexity and variation across 
laminar structures. The authors identified an association between gray matter microstructure in the frontoinsular cortex and life satisfaction, and then they used the twin and sibling design of the dataset to assess the strength of the associations across sibling types. Healthy brain variability is in part due to genetic variation, and identifying a relationship between heritable gray matter microstructure metrics and measures of well being, provide biomarkers of brain health that capture more detailed mechanistic insights into cortical changes occurring throughout the lifespan, than the more commonly assessed macrostructural estimates of cortical thickness and volume.

Lueders and colleagues focus on another young adult population integral to the human lifespan, one that has rarely been assessed with brain imaging - new mothers. In their work, Gray Matter Increases within Subregions of the Hippocampal Complex after Pregnancy, authors dig deeper into the reported changes in the hippocampal volume of women after giving birth to try and localize the specific subfields within the hippocampus where the changes occur. Lueders et al., found lateralized and localized increases in gray matter volumes 4-6 weeks after giving birth as compared to immediately after. This work exemplifies the plasticity of the human brain and the need to better understand not only the individual brain across the lifespan, but also the life cycle. This work, together with that of Luo et al. in this issue, depict how the parent-offspring relationship can have bi-directional associations with brain structure and function.

It is not always evident, however, to what extent longitudinal imaging will provide substantial clinical insights. The documentation of stable metrics is important to avoid unnecessary costs or undue burden on vulnerable clinical populations. In their work, Longitudinal changes of focal cortical glucose hypometabolism in adults with chronic drug resistant temporal lobe epilepsy, Sharpe and colleagues explore whether brain glucose metabolism disruptions-as measured on [18F]-FDG PET reported in adults with drug resistant temporal lobe epilepsy-change over time. This unique sample had originally shown minimal or no initial hypometabolism, contrary to that most often seen in TLE cases. Suspecting hypometabolism would increase with time, the authors used a staggered sampling of longitudinal time intervals ranging from 2 to 95 months, but found no evidence to suggest changes in the relative metabolism of this sample over time in either the ipsilateral or contralateral hemisphere. Their findings highlight the complexity of epilepsy, and suggest a subtype of TLE that does not show progressive increases in hypometabolism with chronic epileptogenesis; results here suggest that additional [18F]-FDG PET scanning on these individuals is not likely to provide additional clinically useful information.

Epilepsy may also be the consequence of brain trauma, but it is not known whether there are specific features of the brain injury that may predict the onset of seizures. In their paper entitled, Distribution and volume analysis of early hemorrhagic contusions by MRI after traumatic brain injury: a preliminary report of the Epilepsy Bioinformatics Study for Antiepileptogenic Therapy (EpiBioS4Rx), La Rocca et al., present preliminary findings from the multicenter observational study of traumatic brain injury of individuals across a wide age range (6-100). They determine the utility of MRI measures derived within 33 days after injury for predicting whether TBI patients developed seizures within two years of injury. Their initial investigation showed neuroimaging features extracted soon after initial insult, specifically the volume of contusions and edema, were indeed different between those who develop posttraumatic epilepsy and those who remain seizure free after two years. MRI biomarkers that may be predictive, independent of age, may be particularly important for predicting future complications of brain injury that may occur at any point along the lifespan.

Capturing the normal population variability of brain integrity with age allows researchers to chart how an individual fits within that norm. The degree to which brain aging appears accelerated or attenuated may reveal information about the individual's brain health. Yet different metrics derived from brain imaging may be sensitive to different pathological alterations within the brain, and provide deeper insights into risk factors for specific disorders. In Age and Sex Effects on Advanced White Matter Microstructure Measures in 15,628 Older Adults: A UK Biobank Study, Lawrence et al. study the age-related trends of white matter microstructure observed in a large population of adults aged 45-80 across a wide range of diffusion imaging metrics. They provide centile curves for all metrics, and show that more advanced multishell metrics are more sensitive to age and sex effects, but that these effects can also be detected with more common diffusion tensor imaging (DTI) metrics in a population of this size.

Environment and lifestyle factors may also contribute to differences in brain aging - identifying modifiable factors, or combinations of factors, that help explain the variability between the lowest and highest centiles, may help reduce the rate of age-related neurodegeneration. Kurth and colleagues study the degree to which meditation may alter the trajectory of brain aging. In Reduced Age-Related Gray Matter Loss in the Subgenual Cingulate Cortex in Long-term Meditators, the authors assess age trajectories in individuals who have been actively meditating for between 4 years and four decades, to age-matched healthy individuals who do not meditate. As expected, older participants had progressively lower gray matter volumes, regardless of meditation group; however, the volume of the subgenual cingulate showed a steeper decline with age in non-meditators compared to meditators. The findings in this cross-sectional study of 
individuals—across a wide age range—-suggest that meditation may have particularly beneficial effects at older ages.

Cross-sectional studies can help understand the patterns of brain aging, yet randomized controlled trials can help establish effects of interventions by monitoring individual level changes. Exercise has been suggested to be a modifiable lifestyle factor that may help preserve brain integrity with age. In the article, Effects of a Physical Activity Intervention on Brain Atrophy in Older Adults at Risk of Dementia: A Randomized Controlled Trial, Sinclair et al. randomized 98 high-risk individuals to a two-year controlled trial with a physical activity intervention. They assessed the rate of tissue loss across the brain using longitudinal voxel-based morphometry. While they found significant reductions in tissue volumes over time, they found no significant differences in the rates of change between the two groups. This work found no evidence to suggest that physical activity interventions may slow the rate of atrophy in older individuals at high risk for dementia.

This issue compiles original investigations of brain structure across the lifespan. Together, we hope these reports of novel findings, as well as null findings, will serve as a valuable guide to neuroimaging across the lifespan.
Acknowledgements The 2020 Pacific Rim New Horizons in Human Brain Imaging: Neuroimaging Across the Lifespan meeting was made possible by the support of our generous funders: Bracco Diagnostics; Siemens Healthcare; The Mark and Mary Stevens Neuroimaging and Informatics Institute; The Laboratory of Neuro Imaging Resource (P41EB015922); The Australian Research Council (ARC) Centre of Excellence for Integrative Brain Function (CE140100007); Monash Biomedical Imaging, Monash University; The Department of Neuroscience, Central Clinical School, Monash University; The Ludmer Centre for Neuroinformatics and Mental Health; The USC Leonard Davis School of Gerontology. The authors acknowledge support from NIH grants P41EB015922, R01MH116147, R01AG059874, Start-up Funds for Leading Talents at Beijing Normal University "Developmental Population Neuroscience", National Basic Science Data Center "Chinese Data-sharing Warehouse for In-vivo Imaging Brain" (NBSDC-DB-15).

Publisher's note Springer Nature remains neutral with regard to jurisdictional claims in published maps and institutional affiliations. 